Research Article

\title{
The Discrete Beverton-Holt Model with Periodic Harvesting in a Periodically Fluctuating Environment
}

\author{
Ziyad AlSharawi and Mohamed Ben Haj Rhouma
}

Department of Mathematics and Statistics, Sultan Qaboos University, Al-Khod, P.O. Box 36, Muscat 123, Oman

Correspondence should be addressed to Ziyad AlSharawi, alsha1zm@squ.edu.om

Received 7 August 2009; Revised 22 November 2009; Accepted 31 January 2010

Academic Editor: Elena Braverman

Copyright (C) 2010 Z. AlSharawi and M. Ben Haj Rhouma. This is an open access article distributed under the Creative Commons Attribution License, which permits unrestricted use, distribution, and reproduction in any medium, provided the original work is properly cited.

\begin{abstract}
We investigate the effect of constant and periodic harvesting on the Beverton-Holt model in a periodically fluctuating environment. We show that in a periodically fluctuating environment, periodic harvesting gives a better maximum sustainable yield compared to constant harvesting. However, if one can also fix the environment, then constant harvesting in a constant environment can be a better option, especially for sufficiently large initial populations. Also, we investigate the combinatorial structure of the periodic sequence of carrying capacities and its effect on the maximum sustainable yield. Finally, we leave some questions worth further investigations.
\end{abstract}

\section{Introduction}

In October 2008, the World Bank and the Food and Agriculture Organization of the United Nations released a study on the economic justification for fisheries reform [1]. The title of the report says it all "The Sunken Billions." The report argues that the sunken $\$ 50$ Billions is a conservative estimate for the losses incurred annually due to carrying business as usual. In general, the study shows a grim picture on the current state of marine fish stocks. The recovery of the sunken billions and wasted harvesting efforts is obviously not an instantaneous process, but rather the product of two main strategies: reducing harvesting efforts and rebuilding of fish stocks. Clearly, the two are very well related; however, a good understanding of theoretical harvesting strategies on population models will go along way in designing an optimal strategy.

There is a wealth of research on the effect of harvesting on the dynamics of populations governed by differential equations. For example, in predator-prey systems, 
constant harvesting can lead to the destabilization of population's equilibria, the creation of limit cycles, different types of bifurcations, catastrophe, and even chaotic behavior [27]. Optimal harvesting for single species has been studied by several authors from different points of view; see for example [8-10] and the references therein. Recently, Braverman and Mamadani [11] considered both autonomous and nonautonomous population models and found that constant harvesting is always superior to impulsive harvesting even though impulsive harvesting can sometimes do as good as constant harvesting. Their results contrast with the results of Ludwig [12] and Xu et al. [13]. For single species, Ludwig [12] studied models with random fluctuations and found that constant effort harvesting does worse than other harvesting strategies. $\mathrm{Xu}$ et al. [13] investigated harvesting in seasonal environments of a population with logistic growth and found that pulse harvesting is usually the dominant strategy and that the yield depends dramatically on the intrinsic growth rate of population and the magnitude of seasonality. Furthermore, for large intrinsic growth rate and small environmental variability, several strategies such as constant exploitation rate, pulse harvest, linear exploitation rate, and time-dependent harvest are quite effective and have comparable maximum sustainable yields. However, for populations with small intrinsic growth rate but subject to large seasonality, none of these strategies is particularly effective, but still pulse harvesting provides the best maximum sustainable yield.

Although the subject of difference equations and discrete models has been flourishing in the past two decades, harvesting in discrete population models is relatively morbid. Constant rate depletion on the discrete Ricker model was studied in [14], where it was shown numerically that populations exhibiting chaotic oscillations are not necessarily vulnerable to extinction. The effect of periodic harvesting on the discrete Ricker model and for a host-parasite model was studied in [15]. The stochastic Beverton-Holt equation with constant and proportional harvesting was studied in [16]. A special type of periodic impulsive harvesting in relation with seasonal environment was also studied in [17]. In [18], AlSharawi and Rhouma examined the effect of harvesting and stocking on competing species governed by a Leslie/Gower model and found that careful harvesting of the dominant species in an exclusive competitive environment can sometimes lead to the survival of the weaker species. More recently, the authors have also studied the Beverton-Holt equation under periodic and conditional harvesting and have found that in a constant capacity environment, constant rate harvesting is the optimal strategy [19].

This paper is a continuation of [19] and it is a modest contribution toward a full understanding of harvesting strategies on discrete population models. We compare the effect of different harvesting strategies in different environments. In particular, we consider and compare the effect of periodic and constant harvesting in both constant and periodic environments in a population governed by the Beverton-Holt model

$$
y_{n+1}=\frac{\mu k_{n} y_{n}}{k_{n}+(\mu-1) y_{n}}, \quad n \in \mathbb{N}:=\{0,1,2, \ldots\}, x_{0} \in \mathbb{R}^{+},
$$

where $\mu>1$ is the population inherent growth rate and $k_{n}$ is the population carrying capacity at time $n$. In our analysis, we focus on the maximum sustainable yield commonly known as the MSY [20]. Despite its disregard to cost, the MSY remains the main criteria for managing populations and avoiding over exploitation. 
The paper is structured as follows: in Sections 2 and 3, we discuss the existence of periodic solutions and the basin of attraction of the stable periodic solution. In Section 4, we address different aspects of constant yield harvesting in periodic environment, then we focus on periodic harvesting in periodic environments and its effect on population's resonance/attenuance. We make a comparison with other harvesting strategies and give a full discussion when $p=2$. Finally, we close the paper with a brief conclusion and a few questions that are worth further investigation.

\section{Preliminary}

In this section, we give a preliminary result that is necessary in our consequent analysis. Assume we have constant harvesting on (1.1) with periodically fluctuating carrying capacities, that is,

$$
x_{n+1}=f_{n}\left(x_{n}\right):=\frac{\mu k_{n} x_{n}}{k_{n}+(\mu-1) x_{n}}-h, \quad k_{n+p}=k_{n}, \quad n \in \mathbb{N},
$$

where $h$ is the constant intensity of harvesting. Since when $h=0, \mu>1$ is a necessary condition for a population to persist, we always assume $\mu>1$. Next, define the maps $F_{0}(x)=I d(x)=x$ and $F_{n}(x)=f_{(n-1) \bmod p}\left(F_{n-1}(x)\right)$ for all $n \in \mathbb{Z}^{+}$. The orbits of (2.1) take the form

$$
\mathcal{O}\left(x_{0}\right)=\left\{x_{0}, F_{1}\left(x_{0}\right), F_{2}\left(x_{0}\right), \ldots, F_{p-1}\left(x_{0}\right), \ldots\right\}
$$

For each $j=0, \ldots, p-1$, define the matrix

$$
B_{j}:=\left[\begin{array}{cc}
k_{j} & \mu-1 \\
-h k_{j} & \mu k_{j}-h(\mu-1)
\end{array}\right]
$$

and consider the operators $T_{j}\left(X_{0}\right)=B_{j} X_{0}$, where $X_{0}=\left[1, x_{0}\right]^{T}$. A simple induction argument shows that orbit (2.2) takes the matrix form

$$
\mathcal{O}^{+}\left(X_{0}\right)=\left\{X_{0}, B_{1} X_{0}, B_{2} X_{0}, \ldots, B_{p-1} X_{0}, \ldots\right\}
$$

where $\boldsymbol{B}_{0}=I$ and $\boldsymbol{B}_{n}=B_{n-1} \boldsymbol{B}_{n-1}$. For more details about this approach, we refer the reader to [19].

Proposition 2.1. Each of the following holds true for (2.1).

(i) If $\operatorname{tr}\left(B_{p}\right)>2 \mu^{p / 2} \prod_{j=0}^{p-1} k_{j}$, then there exist two $p$-cycles; one of them is stable and the other is unstable.

(ii) If $\operatorname{tr}\left(\mathbb{B}_{p}\right)=2 \mu^{p / 2} \prod_{j=0}^{p-1} k_{j}$, then exactly one semistable $p$-cycle exists.

(iii) If $\operatorname{tr}\left(B_{p}\right)<2 \mu^{p / 2} \prod_{j=0}^{p-1} k_{j}$, then there are no periodic solutions and consequently, no population persists. 
Proof. It follows along the same lines as [19, Proof of Theorem 3.3]. Nevertheless, it is enough to observe that the cycles depend on the monotonically increasing function $F_{p}(x)$ in (2.2). $F_{p}(x)$ has two fixed points if and only if the eigenvalues of the matrix $\boldsymbol{B}_{p}$ in (2.4) are distinct real numbers. The eigenvalues of $\boldsymbol{B}_{p}$ are given by

$$
\frac{1}{2} \operatorname{tr}\left(\mathbb{B}_{p}\right) \pm \frac{1}{2} \sqrt{\left(\operatorname{tr}\left(\mathbb{B}_{p}\right)\right)^{2}-4 \operatorname{det}\left(\mathbb{B}_{p}\right)}
$$

and the radicand gives the conditions in (i), (ii), and (iii). If $\bar{y}_{0} \leq \bar{y}_{1}$ are the two fixed points of $F_{p}$, then

$$
\mathcal{O}\left(\bar{y}_{j}\right)=\left\{\bar{y}_{j}, F_{1}\left(\bar{y}_{j}\right), F_{2}\left(\bar{y}_{j}\right), \ldots, F_{p-1}\left(\bar{y}_{j}\right)\right\}, \quad j=0,1,
$$

are the two $p$-cycles of (2.1). Furthermore, since $F_{p}(x)$ is strictly increasing, then the positive equilibrium $\bar{y}_{0}$ is unstable and $\bar{y}_{1}$ is stable. When $\bar{y}_{0}=\bar{y}_{1}$ at $\operatorname{tr}\left(\mathbb{B}_{p}\right)=2 \mu^{p / 2} \prod_{j=0}^{p-1} k_{j}$, we obtain semistability (stability from above only). Because the maps $f_{j}, 0 \leq j \leq p-1$ are continuous, then the cycles of (2.1) inherit the stability of $\bar{y}_{0}$ and $\bar{y}_{1}$ under the monotonic map $F_{p}$. Finally, when $F_{p}$ has no fixed points, then we have a monotonically increasing function below $y=x$ and obviously, orbits go negative in finite time.

Proposition 2.1 shows that a constraint on $h$ is necessary to assure the long-term survival of a population governed by (2.1). The harvesting level reaches its maximum $h_{\max }$ when the $p$-cycle becomes semistable. Thus, we proceed with the assumption that $0 \leq h \leq h_{\max }$, and $h_{\max }$ is the smallest positive solution of the equation $\operatorname{tr}\left(\mathbb{B}_{p}\right)=2 \mu^{p / 2} \prod_{j=0}^{p-1} k_{j}$.

\section{Harvesting Levels and the Basin of Attraction}

It is well known [21, 22] that for $h=0$, system (2.1) has a globally asymptotically stable $p$-cycle, that is, the basin of attraction of the $p$-cycle is $\mathbb{R}^{+}$. In this section, we consider (2.1) with $0<h \leq h_{\max }$ and investigate the basin of attraction of the stable/semistable $p$-cycle. But first, we give a few necessary definitions. A solution of (2.1) is called persistent if the corresponding initial population survives indefinitely. Here, it is worth emphasizing that although one can start iterating (2.1) at any time $n=n_{0}$, time reference is crucial in our analysis, and an initial population is meant $x_{0}$ all the time. A set $\Phi:=\left\{x: x \in \mathbb{R}^{+}\right\}$is persistent if each solution of (2.1) with $x_{0} \in \Phi$ is persistent. We refer those who are interested in reading more about persistence and its significance to $[23,24]$. At a harvesting level $h$, let $\Phi_{h}$ be the largest persistent set, which we simply call the persistent set. Obviously, when $h=0, \Phi_{0}=[0, \infty)$, and $\Phi_{h}$ is empty when $h>h_{\max }$. Thus, a persistent set must contain the basin of attraction of the stable $p$-cycle assured by Proposition 2.1.

Proposition 3.1. Let $0<h \leq h_{\max }$ and let $\left\{\bar{x}_{0, l}, \bar{x}_{1, l}, \ldots, \bar{x}_{p-1, l}\right\}$ be the unstable $p$-cycle. Then $\Phi_{h}=$ $\left[\bar{x}_{0, l}, \infty\right)$. 
Proof. Since $h<h_{\max }$, then the map $F_{p}(x)$ defined in (2.2) has two fixed points, say $\bar{x}_{0, l}$ and $\bar{x}_{0, r}$, where $\bar{x}_{0, l} \leq \bar{x}_{0, r}$. Now, the other elements of the unstable cycle are given by $\bar{x}_{1, l}=f_{0}\left(\bar{x}_{0, l}\right), \bar{x}_{2, l}=f_{1}\left(f_{0}\left(\bar{x}_{0, l}\right)\right)=f_{1}\left(\bar{x}_{1, l}\right), \ldots$ Let $x_{0} \geq \bar{x}_{0, l}$. From the monotonicity of the maps $f_{j}, j=0, \ldots, p-1$, we obtain $x_{n+1} \geq \bar{x}_{n \bmod p, l}>0$ for all $n \in \mathbb{N}$. Thus $\left[\bar{x}_{0, l}, \infty\right) \subseteq \Phi_{h}$. Now, if $x_{0}<\bar{x}_{0, l}$, then $x_{n+1}=F_{p-1}\left(x_{0}\right)$ and the monotonicity of $F_{p-1}$ implies $x_{p+1}<\bar{x}_{0, l}$. For sufficiently large $n, x_{n p+1}=F_{p-1}^{n}\left(x_{0}\right)<0$, which completes the proof.

Proposition 3.2. Suppose that $h \leq(\sqrt{\mu}-1)^{2} k_{j} /(\mu-1)$ for all $j=0, \ldots, p-1$, and let $s_{l, j} \leq s_{r, j}$ be the fixed points of the map $f_{j}$. Then,

$$
\left[\max \left\{s_{l, j}, j=0, \ldots, p-1\right\}, \infty\right) \subseteq \Phi_{h}, \quad \Phi_{h} \subseteq\left[\min \left\{s_{l, j-1}, j=0, \ldots, p-1\right\}, \infty\right)
$$

Proof. Since $h \leq(\sqrt{\mu}-1)^{2} k_{j} /(\mu-1)$ for each $j$, then each map $f_{j}$ has two fixed points $s_{l, j} \leq$ $k /(\sqrt{\mu}+1) \leq s_{r, j}$. Now, trace the iterates of (2.1) for a given initial condition $x_{0}$ to obtain the result.

If we have complete control over the carrying capacities in the $p$-periodic sequence $\left\{k_{j}\right\}$, then Theorem 4.2 shows that we can achieve a maximum harvesting level by taking a constant carrying capacity, that is, $p=1$. However, assume we do not have this absolute power, but we have a flexible control over the periodic permutation of the carrying capacities $\left\{k_{j}\right\}$. In other words, we are considering a difference equation of the form

$$
x_{n+1}=f_{j_{n}}\left(x_{n}\right):=\frac{k_{j_{n}} \mu x_{n}}{k_{j_{n}}+(\mu-1) x_{n}}-h, \quad n \in \mathbb{N},
$$

where $\left\{j_{0}, j_{1}, \ldots, j_{p-1}\right\}$ is a permutation of $\{0,1,2, \ldots, p-1\}$ and $k_{j_{n+p}}=k_{j_{n}}$ for all positive integers $n$. Under these circumstances, we give the next result.

Theorem 3.3. Fix a set of carrying capacities $\left\{k_{0}, k_{1}, \ldots, k_{p-1}\right\}$. All equations of the form (3.2) with permutations $\left(j_{0}, j_{1}, \ldots, j_{p-1}\right)$ in the dihedral group of order $p$ give the same maximum constant harvesting level.

Proof. The maximum harvesting level is the smallest positive solution of the equation

$$
\operatorname{tr}\left(B_{j_{p}} B_{j_{p-1}} \cdots B_{j_{0}}\right)-2 \mu^{p / 2} k_{0} k_{1} \cdots k_{p-1}=0
$$

Now, the elements of the dihedral group $D_{p}$ are rotations and reflections. The rotations are assured by the trivial trace property $\operatorname{tr}\left(B_{j_{i}} B_{j_{k}}\right)=\operatorname{tr}\left(B_{j_{k}} B_{j_{i}}\right)$. For the reflections, we need to show that

$$
\operatorname{tr}\left(B_{j_{p-1}} B_{j_{p-2}} \cdots B_{j_{0}}\right)=\operatorname{tr}\left(B_{j_{0}} B_{j_{1}} \cdots B_{j_{p-1}}\right) .
$$


First, we rewrite the matrix $B_{j_{i}}$ in (2.3) as $B_{j_{i}}=k_{j_{i}} A+(\mu-1) C$, where

$$
A:=\left[\begin{array}{cc}
1 & 0 \\
-h & \mu
\end{array}\right], \quad C:=\left[\begin{array}{cc}
0 & 1 \\
0 & -h
\end{array}\right] .
$$

By simple induction, we can show that

$$
A^{n}=\left[\begin{array}{cc}
1 & 0 \\
-h \frac{\left(\mu^{n+1}-1\right)}{\mu-1} & \mu^{n}
\end{array}\right], \quad C^{n}=(-h)^{n-1} C .
$$

Now, let $p$ be the power set of $\{0,1, \ldots, p-1\}$. We expand the product of the matrices $B_{j_{i}}$ and write

$$
\begin{aligned}
\operatorname{tr}\left(B_{j_{p-1}} B_{j_{p-2}} \cdots B_{j_{0}}\right) & =\sum_{S \in \mathcal{D}}(\mu-1)^{p-|S|} \prod_{i \in S} k_{j_{i}} \operatorname{tr}(D(S)), \\
\operatorname{tr}\left(B_{j_{0}} B_{j_{1}} \cdots B_{j_{p-1}}\right) & =\sum_{S \in \mathcal{D}}(\mu-1)^{p-|S|} \prod_{i \in S} k_{j_{i}} \operatorname{tr}(\widehat{D}(S)),
\end{aligned}
$$

where $|S|$ is the cardinality of the set $S$ and

$$
\begin{aligned}
D(S) & =D_{p-1}(S) D_{p-2}(S) \cdots D_{1}(S) D_{0}(S), \\
\widehat{D}(S) & =D_{0}(S) D_{1}(S) \cdots D_{p-2}(S) D_{p-1}(S), \\
D_{j}(S) & = \begin{cases}A & \text { if } j \in S, \\
C & \text { otherwise. }\end{cases}
\end{aligned}
$$

Now, proving (3.4) is equivalent to proving that

$$
\operatorname{tr}\left(D_{p-1}(S) D_{p-2}(S) \cdots D_{0}(S)\right)=\operatorname{tr}\left(D_{0}(S) D_{1}(S) \cdots D_{p-1}(S)\right)
$$

This is obvious if $S$ is either the empty or the complete set $\{0,1, \ldots, p-1\}$. If $S$ is a nonempty proper subset of $\{0,1, \ldots, p-1\}$, then $D(S)$ contains the product of at least one matrix $A$ and one matrix $C$. Thus, using the rotation property, we can write

$$
\operatorname{tr}(D(S))=\operatorname{tr}\left(A^{\alpha_{1}} C^{\beta_{1}} A^{\alpha_{2}} C^{\beta_{2}} \cdots A^{\alpha_{m}} C^{\beta_{m}}\right)=(-h)^{-m+\sum \beta_{i}} \operatorname{tr}\left(A^{\alpha_{1}} C A^{\alpha_{2}} C \cdots A^{\alpha_{m}} C\right)
$$


for some positive integers $\alpha_{1}, \ldots, \alpha_{m}, \beta_{1}, \ldots, \beta_{m}$ that satisfy $\sum \alpha_{i}=|S|$ and $\sum \beta_{i}=p-|S|$. Since

$$
A^{\alpha_{i}} C=\left[\begin{array}{cc}
0 & 1 \\
0 & \gamma_{\alpha_{i}}
\end{array}\right], \quad \gamma_{\alpha_{i}}=-\frac{\left(\mu^{\alpha_{i}+1}-1\right) h}{\mu-1}
$$

then

$$
\operatorname{tr}(D(S))=(-h)^{-m+\sum \beta_{i}} \prod_{i=1}^{m} \gamma_{\alpha_{i}}
$$

On the other hand,

$$
\begin{aligned}
\operatorname{tr}(\widehat{D}(S)) & =\operatorname{tr}\left(C^{\beta_{m}} A^{\alpha_{m}} \cdots C^{\beta_{2}} A^{\alpha_{2}} C^{\beta_{1}} A^{\alpha_{1}}\right) \\
& =\operatorname{tr}\left(A^{\alpha_{m}} \cdots C^{\beta_{2}} A^{\alpha_{2}} C^{\beta_{1}} A^{\alpha_{1}} C^{\beta_{m}}\right) \\
& =(-h)^{-m+\sum \beta_{i}} \operatorname{tr}\left(A^{\alpha_{m}} C \cdots A^{\alpha_{2}} C A^{\alpha_{1}} C\right) \\
& =(-h)^{-m+\sum \beta_{i}} \prod_{i=1}^{m} \gamma_{\alpha_{i}},
\end{aligned}
$$

which completes the desired proof.

Next, we give the polynomials $\operatorname{tr}\left(\mathcal{B}_{p}\right)$ for $p=2,3,4$, whose lowest positive root gives the maximal constant harvesting level in a periodic environment, then we give an illustrative example.

$$
\begin{aligned}
\operatorname{tr}\left(\mathbb{B}_{2}\right)= & (1-\mu)^{2} h^{2}-(\mu-1)(\mu+1)\left(k_{0}+k_{1}\right) h+k_{1} k_{0}\left(\mu^{2}+1\right), \\
\operatorname{tr}\left(\mathbb{B}_{3}\right)= & -(\mu-1)^{3}(h)^{3}+(\mu+1)(\mu-1)^{2} h^{2}\left(\sum_{j=0}^{2} k_{j}\right) \\
& -h\left(\left(\mu^{3}-1\right) \sum_{i=0}^{1} \sum_{j=i+1}^{2} k_{i} k_{j}\right)+k_{2} k_{1} k_{0}\left(\mu^{3}+1\right), \\
\operatorname{tr}\left(\mathbb{B}_{4}\right)= & (\mu-1)^{4} h^{4}-(\mu+1)(\mu-1)^{3} h^{3}\left(\sum_{j=0}^{3} k_{j}\right) \\
& +(\mu-1)^{2} h^{2}\left(\mu\left(k_{0} k_{2}+k_{1} k_{3}\right)+\left(\mu^{2}+\mu+1\right) \sum_{i=0}^{2} \sum_{j=i+1}^{3} k_{j} k_{i}\right) \\
& -\left(\mu^{4}-1\right) k_{0} k_{1} k_{2} k_{3} h\left(\sum_{j=0}^{3} \frac{1}{k_{j}}\right)+\left(\mu^{4}+1\right) k_{0} k_{1} k_{2} k_{3} .
\end{aligned}
$$


Example 3.4. (i) Consider the case $p=2, k_{0}=1, k_{1}=4$, and $\mu=4$. Then the value of $h_{\max }=1 / 6(25-\sqrt{481})$ and the semistable 2-cycle is $\left\{\bar{y}_{0}=1 / 30(\sqrt{481}-1), \bar{y}_{1}=\right.$ $2 / 15(\sqrt{481}-19)\}$ with the interval $[1 / 30(\sqrt{481}-1), \infty)$ as the basin of attraction. Changing the order of the carrying capacities to $k_{0}=4, k_{1}=1$, does not change the value of $h_{\max }$, but it does in return extend the basin of attraction to $[2 / 15(\sqrt{481}-$ 19), $\infty)$. In fact, for constant harvesting in periodic environment with $p=2$, the order of carrying capacities does not affect $h_{\max }$, but $k_{0} \geq k_{1}$ will enlarge the basin of attraction.

(ii) For $p=3$, the order of $\left\{k_{j}\right\}$ does not change $h_{\max }$. This is a little striking since in the absence of harvesting, the order of $\left\{k_{j}\right\}$ does change the average population. In fact, if $\mu=4, k_{0}=1 / 2, k_{1}=2$, and $k_{2}=30$ and in the absence of harvesting, the average population is $\bar{y}=2.195$ which is $31.34 \%$ more than the average population of $\bar{y}=1.671$ obtained if the carrying capacities were presented in the order $k_{0}=30$, $k_{1}=2$, and $k_{2}=1 / 2$. The difference between the two populations is actually as high as $83 \%$ if $\mu=16$.

(iii) For $p=4$, there are 24 permutations of $\left\{k_{j}\right\}$ but the value of $\operatorname{tr}\left(\mathcal{B}_{4}\right)$ can only take three possible values. For each of these values, there corresponds a value of $h_{\max }$. For instance, if

$$
\left\{k_{j}\right\}=\left\{j+10\left(1+(-1)^{j}\right): j=0,1,2,3\right\},
$$

then $\left(k_{0}, k_{1}, k_{2}, k_{3}\right)=(20,1,22,3),(3,22,1,20)$ and their cyclic permutations give $h_{\max 3}=0.932825,\left(k_{0}, k_{1}, k_{2}, k_{3}\right)=(20,1,3,22),(22,3,1,20)$ and their cyclic permutations give $h_{\max 2}=0.892442$, and $\left(k_{0}, k_{1}, k_{2}, k_{3}\right)=(20,3,1,22),(22,1,3,20)$ and their cyclic permutations give $h_{\max 1}=0.892846$. Notice that the difference between the two extremes is about $5 \%$.

The next result shows which permutation would maximize the harvesting level for some values of $p$.

Theorem 3.5. Consider (3.2) and assume the initial population is sufficiently large. Without loss of generality, let $k_{0} \leq k_{1} \leq \cdots \leq k_{p-1}$. Each of the following holds true.

(i) For $p=2$ or 3 , a permutation of the carrying capacities does not change the maximum harvesting level.

(ii) For $p=4$, we can achieve three different levels of maximum harvesting through permutations of the carrying capacities. In particular, $\left(j_{0}, j_{1}, j_{2}, j_{3}\right)=(0,2,1,3)$ or $(3,1,2,0)$ and their cyclic permutations give the largest, and $\left(j_{0}, j_{1}, j_{2}, j_{3}\right)=(3,2,0,1)$ or $(1,0,2,3)$ and their cyclic permutations give the smallest.

(iii) For $p=5$, we can achieve twelve different levels of maximum harvesting through permutations of the carrying capacities. In particular, $\left(j_{0}, j_{1}, j_{2}, j_{3}\right)=(1,2,3,0,4)$ or $(4,0,3,2,1)$ and their cyclic permutations give the largest, and $\left(j_{0}, j_{1}, j_{2}, j_{3}\right)=(3,1,0,2,4)$ or $(4,2,0,1,3)$ and their cyclic permutations give the smallest. 
Proof. Since the maximum harvesting level for each permutation $\left(j_{0}, j_{1}, \ldots, j_{p-1}\right)$ is achieved at

$$
\operatorname{tr}\left(B_{j_{p-1}} B_{j_{p-2}} \cdots B_{j_{1}} B_{j_{0}}\right)=2 \sqrt{\operatorname{det}\left(B_{j_{p-1}} B_{j_{p-2}} \cdots B_{j_{1}} B_{j_{0}}\right)}=2 \mu^{p / 2} \prod_{i=0}^{p-1} k_{i}
$$

then we need to investigate the minimum positive value of $h$ that satisfies this equation. (i) follows straight from the expressions of $\operatorname{tr}\left(\mathbb{B}_{2}\right)$ and $\operatorname{tr}\left(\mathbb{B}_{3}\right)$. To prove (ii), classify the 4 ! elements of the permutation group into three subgroups, each of which is isomorphic to the dihedral group of order 4. Now, Theorem 3.3 says that it is possible to obtain three different values of $h_{\max }$. More specifically, $\left(j_{0}, j_{1}, j_{2}, j_{3}\right)=(3,2,0,1)$ or $(1,0,2,3)$ and their cyclic permutations give the same maximum harvesting level, say $h_{\max 1}$. Similarly, $\left(j_{0}, j_{1}, j_{2}, j_{3}\right)=$ $(0,1,2,3)$ or $(3,2,1,0)$ and their cyclic permutations give $h_{\max 2},\left(j_{0}, j_{1}, j_{2}, j_{3}\right)=(0,2,1,3)$ or $(3,1,2,0)$ and their cyclic permutations give $h_{\max 3}$. Now, we proceed to show that $h_{\max 1} \leq h_{\max 2} \leq h_{\max 3}$. Define

$$
\begin{aligned}
& q_{1}(h):=\operatorname{tr}\left(B_{3} B_{2} B_{0} B_{1}\right)-2 \mu^{2} k_{3} k_{2} k_{1} k_{0}, \\
& q_{2}(h):=\operatorname{tr}\left(B_{3} B_{2} B_{1} B_{0}\right)-2 \mu^{2} k_{3} k_{2} k_{1} k_{0}, \\
& q_{3}(h):=\operatorname{tr}\left(B_{3} B_{0} B_{2} B_{1}\right)-2 \mu^{2} k_{3} k_{2} k_{1} k_{0},
\end{aligned}
$$

then $q_{i}(0)>0$ and $q_{i}\left(h_{\max i}\right)=0$ for $i=1,2,3$. Furthermore, straightforward computations show that

$$
\begin{aligned}
& q_{3}(h)=q_{2}(h)+\mu(\mu-1)^{2}\left(k_{1}-k_{2}\right)\left(k_{0}-k_{3}\right) h^{2}, \\
& q_{3}(h)=q_{1}(h)+\mu(\mu-1)^{2}\left(k_{1}-k_{3}\right)\left(k_{0}-k_{2}\right) h^{2}, \\
& q_{1}(h)=q_{2}(h)+\mu(\mu-1)^{2}\left(k_{3}-k_{2}\right)\left(k_{0}-k_{1}\right) h^{2} .
\end{aligned}
$$

Now, $q_{1}\left(h_{\max 2}\right) \leq 0$ implies that $h_{\max 1} \leq h_{\max 2}, q_{1}\left(h_{\max 3}\right) \leq 0$ implies that $h_{\max 1} \leq h_{\max 3}$ and $q_{2}\left(h_{\max 3}\right) \leq 0$ implies that $h_{\max 2} \leq h_{\max 3}$. The proof of (iii) is computational and too long; however, it follows along the same lines as the proof of (ii), and thus, we omit it.

\section{Periodic Harvesting in a Periodic Environment}

In this section, we consider

$$
x_{n+1}=f_{n}\left(x_{n}\right):=\frac{k_{n} \mu x_{n}}{k_{n}+(\mu-1) x_{n}}-h_{n}, \quad k_{n+p}=k_{n}, \quad h_{n+p}=h_{n}, \quad n \in \mathbb{N} .
$$

Observe that if $h_{n}=h$ for all $n$, then we have the constant yield harvesting. Thus we discuss the constant yield harvesting first followed by the more general periodic case, then we discuss resonance and attenuance. Finally, for the sake of concreteness, we focus on the specific case $p=2$. 


\subsection{Constant Yield Harvesting in a Periodic Environment}

We force $h_{n}=h$ in (4.1) to obtain (2.1). Observe that $f_{j}(x)$ is asymptotic to $\mu k_{j} / \mu-1$. So, it is obvious that $0 \leq h \leq h_{\max }<\min \left\{(\mu / \mu-1) k_{j}, j=0,1, \ldots, p-1\right\}$, where $h_{\max }$ is a threshold level of harvesting that needs to be investigated. The next result gives an upper bound on the maximal harvesting level $h_{\max }$.

Proposition 4.1. Consider (2.1), then

$$
h_{\max }<\min \left\{\left(\sum_{j=0}^{p-1} \mu^{j}\right)\left(\sum_{j=0}^{p-1} \frac{\mu^{j}}{k_{j+i \bmod p}}\right)^{-1}, i=0,1, \ldots, p-1\right\} .
$$

Proof. The set on the right-hand side of the inequality is the stable cycle at zero harvesting level.

By now, it is well known that periodic environment does not enhance populations governed by the Beverton-Holt model with constant growth rate and periodic capacity [21, $22,25,26]$. This suggests that periodic environment has a negative impact on the maximum harvesting level. Indeed, we have the following result.

Theorem 4.2. Consider (2.1); then the maximum harvesting level $h_{\max }$ in a periodic environment is less than the maximum harvesting level in a constant environment with $k=k_{a v}:=1 / p \sum_{j=0}^{p-1} k_{j}$.

Proof. Let $\left\{\bar{x}_{0}, \bar{x}_{1}, \ldots, \bar{x}_{p-1}\right\}$ be the semistable $p$-cycle assured at the maximum harvesting level $h_{\text {max }}$. From (2.1), we obtain

$$
\sum_{j=0}^{p-1} x_{j}=-p h_{\max }+\sum_{j=0}^{p-1} \frac{k_{j} \mu x_{j}}{k_{j}+(\mu-1) x_{j}}
$$

and thus

$$
h_{\max }=\frac{1}{p} \sum_{j=0}^{p-1}\left(\frac{k_{j} \mu x_{j}}{k_{j}+(\mu-1) x_{j}}-x_{j}\right) .
$$

Since the map $h_{j}(t)=\left(k_{j} \mu t /\left(k_{j}+(\mu-1) t\right)\right)-t, t>0$ has absolute maximum at $t=((\sqrt{\mu}-$ 1) $/ \mu-1) k_{j}$, then

$$
h_{\max }<\frac{1}{p} \frac{(\sqrt{\mu}-1)^{2}}{(\mu-1)} \sum_{j=0}^{p-1} k_{j}=\frac{(\sqrt{\mu}-1)^{2}}{(\mu-1)} k_{a v} .
$$

The right-hand side of the inequality is the maximum harvesting level at the constant carrying capacity $k=k_{a v}$, which completes the proof. 


\subsection{The General Case}

By considering the matrix of (2.3) to be

$$
B_{j}:=\left[\begin{array}{cc}
k_{j} & \mu-1 \\
-h_{j} k_{j} & \mu k_{j}-h_{j}(\mu-1)
\end{array}\right],
$$

Proposition 2.1 continues to hold with the exception that cycles period may not be minimal, that is, the cycle's period could be a divisor of $p$. This is due to the freedom in the two parameters $k_{j}$ and $h_{j}$. For instance, consider $p=\mu=4$ and

$$
k_{0}=\frac{1}{2}(9+3 \sqrt{17}), \quad k_{1}=9, \quad k_{3}=\frac{1}{4}(15+3 \sqrt{57}), \quad k_{4}=\frac{1}{5}(3+2 \sqrt{21}), \quad h_{j}=\frac{3\left(k_{j}-1\right)}{k_{j}+3} .
$$

In this case, $\bar{x}=1$ is an equilibrium point and $\left\{\bar{x}_{j}=j+2\right\}_{j=0}^{3}$ is a 4-cycle. Furthermore, $[1, \infty)$ is the persistent set. For more details about the structure of periodic solutions in periodic discrete systems, we refer the reader to $[27,28]$.

In a constant capacity environment with $k=k_{a v}=1 / p \sum_{j=0}^{p-1} k_{i}$, the maximum constant harvesting is $h_{\max }=\left((\sqrt{\mu}-1)^{2} / \mu-1\right) k_{a v}$. The following theorem indicates that periodic harvesting in a periodic environment gives an average harvest rate less than $h_{\max }$.

Theorem 4.3. Consider $h_{a v}$ to be the average of the maximum harvesting levels in (4.1). Then

$$
h_{a v}:=\frac{1}{p} \sum_{j=0}^{p-1} h_{j}<h_{\max }:=\frac{(\sqrt{\mu}-1)^{2}}{\mu-1} k_{a v} .
$$

Proof. If (4.1) has no periodic solution, then no population persists. So, let $\left\{x_{j}\right\}_{0}^{p-1}$ be a periodic solution of period $p$ (not necessarily minimal). Now, use the same argument as in the proof of Theorem 4.2 to obtain the result.

Despite the inferiority of $h_{a v}$ as shown in Theorem 4.3, one cannot underestimate the flexibility of periodic harvesting in terms of harvesting efforts and the effect on populations. Let $0<\beta \leq k_{j}$ for all $0 \leq j<p$ and take

$$
h_{j}=\frac{\beta(\mu-1)\left(k_{j}-\beta\right)}{k_{j}+(\mu-1) \beta}
$$

then $\bar{x}=\beta$ is an equilibrium of (4.1). Furthermore, when $\beta$ is unstable, that is,

$$
\prod_{j=0}^{p-1}\left(1+\frac{\mu-1}{k_{j}} \beta\right) \leq \mu^{p / 2}
$$

then $[\beta, \infty)$ is the persistent set, which gives us the advantage of controlling the persistent set for the benefit of low-level populations. 
Theorems 4.2 and 4.3 along with the results of [19] prove that in order to maximize harvesting, when given a choice of environment and type of harvesting, constant harvesting in constant environment is superior. Suppose we are given a choice between two options: (1) periodically harvesting in a constant capacity environment and (2) constantly harvesting in a periodic environment. The next theorem asserts that option (1) can be better if done carefully.

Theorem 4.4. Let $h_{\max }$ be the maximum harvesting level that can be achieved with periodic carrying capacity $\left\{k_{0}, k_{1}, \ldots, k_{p-1}\right\}$. We can find harvesting quotas $h_{0}, h_{1}, \ldots, h_{p-1}$ in a constant environment with $k_{a v}=1 / p \sum k_{j}$ such that $1 / p \sum h_{j}>h_{\max }$.

Proof. Take the maximum harvesting level $h^{*}$ in a constant environment with $k_{a v}$, then $h^{*}>$ $h_{\max }$ by Theorem 4.2. Now take $h_{0}, h_{1}, \ldots, h_{p-1} \in\left[h^{*}-\epsilon, h^{*}+\epsilon\right]$ for sufficiently small $\epsilon$ to achieve the required task.

\subsection{Resonance and Attenuance}

It is well known $[21,25]$ that populations governed by the periodic Beverton-Holt model

$$
x_{n+1}=\frac{\mu k_{n} x_{n}}{k_{n}+(\mu-1) x_{n}}
$$

exhibit attenuance, that is, the average of the stable cycle is less than the stable equilibrium in the deterministic Beverton-Holt model with carrying capacity equaling the average of the carrying capacities in (4.11). AlSharawi and Rhouma [19] also found that periodic harvesting in a deterministic environment

$$
x_{n+1}=\frac{\mu k x_{n}}{k+(\mu-1) x_{n}}-h_{n}
$$

forces populations governed by the Beverton-Holt model to attenuate. This discussion motivates us to discuss whether populations governed by (4.1) exhibit attenuance too. Indeed, our next theorem shows that the Cushing-Henson Conjecture [21, 22, 25, 26, 29] is valid for (4.1).

Theorem 4.5. Populations governed by (4.1) exhibit attenuance.

To simplify the proof, let us give some simple facts. For constant harvesting in a constant environment with $k=k_{a v}=\sum_{j=0}^{p-1} k_{j}$, simple computations show that the stable (or semistable) equilibrium $\bar{x}_{2}$ of

$$
x_{n+1}=\frac{\mu k_{a v} x_{n}}{k_{a v}+(\mu-1) x_{n}}-h_{a v}
$$

exists when $h_{a v} \leq((\sqrt{\mu}-1) /(\sqrt{\mu}+1)) k_{a v}$ and satisfies the inequality

$$
\frac{\sqrt{\mu}-1}{\mu-1} k_{a v} \leq \bar{x}_{2}<k_{a v}
$$

The next lemma [30] is a simple generalization of Jensen's inequality. 
Lemma 4.6. Let $g: \mathbb{R}^{+} \rightarrow \mathbb{R}$ be a strictly concave function, and let $f: \mathbb{R}^{+2} \rightarrow \mathbb{R}$ be defined as $f(x, k)=k g(x / k)$, then

$$
\sum_{j=1}^{n} f\left(x_{j}, k_{j}\right) \leq f\left(\sum_{j=1}^{n} x_{j}, \sum_{j=1}^{n} k_{j}\right) .
$$

Now, we are ready to prove Theorem 4.5.

Proof of Theorem 4.5. If $\operatorname{tr}\left(\boldsymbol{B}_{p}\right)<2 \mu^{p / 2} \prod_{j=0}^{p-1} k_{j}$, where $\boldsymbol{B}_{p}$ is as defined in (2.4) and $B_{j}$ is as defined in (4.6), then no population will persist and thus, we have trivial attenuance. So, we assume $\operatorname{tr}\left(B_{p}\right) \geq 2 \mu^{p / 2} \prod_{j=0}^{p-1} k_{j}$ and consider $x_{a v}$ to be the average of the stable (or semistable) $p$-cycle (the period is not necessarily minimal) of (4.1). From (4.1), we obtain

$$
h_{a v}=\frac{1}{p} \sum_{j=0}^{p-1} \frac{x_{j}(\mu-1)\left(k_{j}-x_{j}\right)}{k_{j}+(\mu-1) x_{j}} .
$$

Use Lemma 4.6 to obtain

$$
h_{a v} \leq \frac{(\mu-1) x_{a v}\left(k_{a v}-x_{a v}\right)}{k_{a v}+(\mu-1) x_{a v}} \leq \frac{\sqrt{\mu}-1}{\sqrt{\mu}+1} k_{a v} .
$$

Thus, $\bar{x}_{2}$ exists in (4.13) and

$$
h_{a v}=\frac{\bar{x}_{2}(\mu-1)\left(k_{a v}-\bar{x}_{2}\right)}{k_{a v}+(\mu-1) \bar{x}_{2}} .
$$

From Inequality (4.17) and (4.18), we obtain

$$
\frac{\bar{x}_{2}\left(k_{a v}-\bar{x}_{2}\right)}{k_{a v}+(\mu-1) \bar{x}_{2}} \leq \frac{x_{a v}\left(k_{a v}-x_{a v}\right)}{k_{a v}+(\mu-1) x_{a v}}
$$

Now, use Inequality (4.14) and the fact that $h(t):=t\left(k_{a v}-t\right) /\left(k_{a v}+(\mu-1) t\right)$ is decreasing on the interval $\left[((\sqrt{\mu}-1) /(\mu-1)) k_{a v}, \infty\right)$ to obtain $x_{a v} \leq \bar{x}_{2}$.

\subsection{The Case $p=2$}

Let us focus on (4.1) with $p=2$.

Lemma 4.7. Consider periodic harvesting in a periodic environment with $p=2$. Then

$$
h_{j}<\frac{(\mu-1) k_{0} k_{1}}{\mu k_{j+1}+k_{j}}<k_{j} .
$$


Proof. Solve $\operatorname{tr}\left(B_{1} B_{0}\right)-2 \mu k_{0} k_{1}=0$ for $h_{j}$ to obtain

$$
(\mu-1) k_{0} k_{1}=h_{0} k_{0}+h_{1} k_{1}+\mu\left(h_{0} k_{1}+h_{1} k_{0}\right)+h_{0} h_{1}(\mu-1)
$$

In particular, if both $h_{0}$ and $h_{1}$ are nonnegative then

$$
h_{0}<\frac{(\mu-1) k_{0} k_{1}}{k_{0}+\mu k_{1}} \leq k_{0}, \quad h_{1}<\frac{(\mu-1) k_{0} k_{1}}{k_{1}+\mu k_{0}} \leq k_{1} .
$$

Theorem 4.8. Consider (4.1) with $p=2$.

(i) If $k_{1} \geq \sqrt{\mu} k_{0}$, then $h_{0}=0$ and $h_{1}=(\mu-1) k_{0} k_{1} /\left(k_{0} \mu+k_{1}\right)$ give the maximum harvesting average. Moreover, the 2 -cycle is

$$
\left\{\bar{x}_{0}, \bar{x}_{1}\right\}=\left\{\frac{k_{1} k_{0}}{k_{0} \mu+k_{1}}, \frac{k_{0} k_{1}}{k_{1}+k_{0}}\right\}
$$

(ii) If $k_{0}<\sqrt{\mu} k_{1}<\mu k_{0}$, then

$$
h_{0}=\frac{k_{0} \sqrt{\mu}-k_{1}}{\sqrt{\mu}+1}, \quad h_{1}=\frac{k_{1} \sqrt{\mu}-k_{0}}{\sqrt{\mu}+1}
$$

give the maximum harvesting average. Moreover, the 2-cycle is

$$
\left\{\bar{x}_{0}, \bar{x}_{1}\right\}=\left\{\frac{k_{0}}{\sqrt{\mu}+1}, \frac{k_{1}}{\sqrt{\mu}+1}\right\} .
$$

(iii) If $\sqrt{\mu} k_{1} \leq k_{0}$, then $h_{1}=0$ and $h_{0}=(\mu-1) k_{0} k_{1} /\left(k_{1} \mu+k_{0}\right)$ give the maximum harvesting average. Moreover, the 2-cycle is

$$
\left\{\bar{x}_{0}, \bar{x}_{1}\right\}=\left\{\frac{k_{1} k_{0}}{k_{0}+k_{1}}, \frac{k_{0} k_{1}}{\mu k_{1}+k_{0}}\right\}
$$

Furthermore, the persistent set in each case is $\left[\bar{x}_{0}, \infty\right)$.

Proof. To prove (ii), use Lagrange multipliers to maximize the average of $h_{0}$ and $h_{1}$ subject to the constraint $\operatorname{tr}\left(B_{1} B_{0}\right)=2 \mu k_{0} k_{1}$, then use the known values of $h_{0}$ and $h_{1}$ to find the 2-cycle. The values of $h_{0}$ and $h_{1}$ in (i) follow from (ii) and the extra constraints on $h_{0}$ and $h_{1}$ as given in Lemma 4.7, then use the known values of $h_{0}$ and $h_{1}$ to find the 2-cycle. (iii) follows from (i) by swapping the order of $k_{0}$ and $k_{1}$.

Next, we make comparison between the harvesting strategies. 
Theorem 4.9. Consider $p=2$ and assume the initial population is sufficiently large. Periodic harvesting in a periodic environment gives larger harvesting average compared to constant harvesting in a periodic environment.

Proof. Consider $h_{0}=h_{1}=h_{\max }$ and solve $\operatorname{tr}\left(B_{1} B_{0}\right)=2 \mu k_{0} k_{1}$ for $h_{\max }$ to find

$$
h_{\max }=\frac{k_{1}+k_{0}}{2} \frac{\mu+1}{\mu-1}-\frac{\sqrt{(\mu+1)^{2}\left(k_{1}-k_{0}\right)^{2}+16 \mu k_{0} k_{1}}}{2(\mu-1)} .
$$

Now, compare $h_{\max }$ with the average of $h_{0}$ and $h_{1}$ from Theorem 4.8. If $k_{1} \geq \sqrt{\mu} k_{0}$, then $h_{a v}=(\mu-1) k_{0} k_{1} / 2\left(k_{0} \mu+k 1\right)$ and $h_{a v}-h_{\max }=0$ when

$$
k_{1}=\frac{1}{4}\left(-(\mu-1) \pm \sqrt{(\mu-1)^{2}+16 \mu}\right) k_{0}, 0
$$

However, since

$$
\frac{1}{4}\left(-(\mu-1)+\sqrt{(\mu-1)^{2}+16 \mu}\right) k_{0}<\sqrt{\mu} k_{0}
$$

then $h_{a v}-h_{\max }$ does not change sign for all $k_{1}>\sqrt{\mu} k_{0}$. Furthermore, fixed values of $\mu, k_{0}, k_{1}$ show that $h_{a v}-h_{\max }>0$. If $k_{0}<\sqrt{\mu} k_{1}<\mu k_{0}$, then $h_{a v}=\left(\left(k_{0}+k_{1}\right) / 2\right)((\sqrt{\mu}-1) /(\sqrt{\mu}+1))$, and $h_{a v}-h_{\max }=0$ if and only if $k_{0}=k_{1}$. Assuming $k_{0} \neq k_{1}$, we obtain $h_{a v}-h_{\max }>0$.

Now, let us present a detailed comparison in the following illustrative examples.

Example 4.10. Consider

$$
\begin{aligned}
x_{n+1} & =\frac{\mu x_{n}}{1+(\mu-1) x_{n}}-h, \\
y_{n+1} & =\frac{\mu\left(1+(-1)^{n} k\right) y_{n}}{\left(1+(-1)^{n} k\right)+(\mu-1) y_{n}}-h, \quad 0<k<1, \\
z_{n+1} & =\frac{\mu\left(1+(-1)^{n} k\right) z_{n}}{\left(1+(-1)^{n} k\right)+(\mu-1) z_{n}}-h_{n}, \quad 0<k<1 .
\end{aligned}
$$

The first equation is for constant harvesting in a constant environment, (4.31) is for constant harvesting in a periodic environment, and (4.32) is for periodic harvesting in a periodic environment. Notice that we are taking $k=1$ in (4.30), while in both (4.31) and (4.32) and for comparison reasons, we assumed that the carrying capacities alternate periodically between the values $k_{0}:=(1+k)$ and $k_{1}:=(1-k)$ to obtain the average $k_{a v}=1$. Let $h_{c c}, h_{p c}$ and $h_{p p}$ be respectively the maximal harvesting levels for equations (4.30), (4.31), and (4.32). Straightforward computations give

$$
h_{p c} \leq h_{p p} \leq h_{c c}
$$


where

$$
\begin{aligned}
& h_{p c}=\frac{(\mu+1)-\sqrt{(\mu-1)^{2} k^{2}+4 \mu}}{\mu-1}, \\
& h_{c c}=\frac{(\sqrt{\mu}-1)^{2}}{\mu-1}, \\
& h_{p p}= \begin{cases}h_{c c} & \text { if } k \leq \frac{(\sqrt{\mu}-1)^{2}}{\mu-1}, \\
\frac{(\mu-1)\left(1-k^{2}\right)}{2[(\mu+1)+k(1-\mu)]} & \text { if } \frac{(\sqrt{\mu}-1)^{2}}{\mu-1}<k<1 .\end{cases}
\end{aligned}
$$

Example 4.11. In each of the following cases, consider $p=2$.

(i) Consider periodic harvesting in a periodic environment with $\mu=4, k_{0}=3, k_{1}=$ $5, h_{0}=1 / 3$, and $h_{1}=7 / 3$. Then

(a) the average harvesting is $1 / 2\left(h_{0}+h_{1}\right)=4 / 3$;

(b) the 2-cycle is $\left\{\bar{x}_{0}, \bar{x}_{1}\right\}=\{1,5 / 3\}$, which has an average of $4 / 3$;

(c) the persistent set is $\left[\bar{x}_{0}, \infty\right)=[1, \infty)$.

(ii) Consider constant harvesting in a periodic environment with $\mu=4, k_{0}=3, k_{1}=5$. Then

(a) The average harvesting is $h_{\max }=1 / 3(20-\sqrt{265}) \approx 1.240$;

(b) The 2-cycle is

$$
\left\{\bar{x}_{0}, \bar{x}_{1}\right\}=\left\{\frac{-5}{8}+\frac{\sqrt{265}}{8}, \frac{-55}{24}+\frac{5 \sqrt{265}}{24}\right\},
$$

which has an average of $(-35 / 24)+(\sqrt{265} / 6) \approx 1.255$;

(c) The persistent set is $\left[\bar{x}_{0}, \infty\right) \approx[1.410, \infty)$.

\section{Conclusion and Discussion}

In a previous paper [19], we have established that for the deterministic Beverton-Holt model, constant harvesting is superior to both periodic and conditional harvestings when the maximum sustainable yield is taken as the management objective, and when the initial population is sufficiently large. In this paper, we obtained the following.

(i) Constant harvesting in a constant environment is "better" than constant harvesting in a periodic environment (Theorem 4.2 ).

(ii) Constant harvesting in a constant environment is "better" than periodic harvesting in a periodic environment (Theorem 4.3 ). However, at least in the case $p=2$ and for some range of the parameters, careful periodic harvesting can lead to the same yield as the optimal constant harvesting.

(iii) Periodic harvesting in a periodic environment is "better" than constant harvesting in a periodic environment. 
Finally, this study left us with few questions that deserve further investigations.

Question 1. Fix a set of carrying capacities $\left\{k_{0}, k_{1}, \ldots, k_{p-1}\right\}$, and consider all permutations of $\left(k_{0}, k_{1}, \ldots, k_{p-1}\right)$ in (3.2). According to the Theorem 3.3, we obtain $1 / 2(p-1)$ ! values

for $h_{\max }$, and Theorem 3.5 characterizes those values for $p=2,3,4$, and 5. Complete the characterization for general $p$.

Question 2. Consider (4.1) and let $H:=\left\{h_{0}, h_{1}, \ldots, h_{p-1}\right\}$ be a set of harvesting quotas that give a nonempty persistent set. Which permutation of $H$ would enlarge the persistent set?

Question 3. Generalize the results of this study to the case where the inheritance growth rate $\mu$ is nonconstant.

\section{Acknowledgments}

The authors would like to thank the anonymous referee for his/her valuable suggestions. This work is supported by SQU internal Grant DVC/PSR/2009/36.

\section{References}

[1] K. Kelleher, R. Willmann, and R. Arnason, The Sunken Billions, the Economic Justification for Fisheries Reform, The World Bank, Washington, DC, USA, 2009.

[2] C. Azar, J. Holmberg, and K. Lindgren, "Stability analysis of harvesting in a predator-prey model," Journal of Theoretical Biology, vol. 174, no. 1, pp. 13-19, 1995.

[3] F. Brauer and A. C. Soudack, "Stability regions and transition phenomena for harvested predator-prey systems," Journal of Mathematical Biology, vol. 7, no. 4, pp. 319-337, 1979.

[4] F. Brauer and A. C. Soudack, "Stability regions in predator-prey systems with constant-rate prey harvesting," Journal of Mathematical Biology, vol. 8, no. 1, pp. 55-71, 1979.

[5] G. Dai and M. Tang, "Coexistence region and global dynamics of a harvested predator-prey system," SIAM Journal on Applied Mathematics, vol. 58, no. 1, pp. 193-210, 1998.

[6] M. R. Myerscough, B. F. Gray, W. L. Hogarth, and J. Norbury, "An analysis of an ordinary differential equation model for a two-species predator-prey system with harvesting and stocking," Journal of Mathematical Biology, vol. 30, no. 4, pp. 389-411, 1992.

[7] D. Xiao and S. Ruan, "Bogdanov-Takens bifurcations in predator-prey systems with constant rate harvesting," in Differential Equations with Applications to Biology (Halifax, NS, 1997), vol. 21 of Fields Institute Communications, pp. 493-506, American Mathematical Society, Providence, RI, USA, 1999.

[8] S. Tang and L. Chen, "The effect of seasonal harvesting on stage-structured population models," Journal of Mathematical Biology, vol. 48, no. 4, pp. 357-374, 2004.

[9] Y. Xiao, D. Cheng, and H. Qin, "Optimal impulsive control in periodic ecosystem," Systems E Control Letters, vol. 55, no. 7, pp. 558-565, 2006.

[10] X. Zhang, Z. Shuai, and K. Wang, "Optimal impulsive harvesting policy for single population," Nonlinear Analysis: Real World Applications, vol. 4, no. 4, pp. 639-651, 2003.

[11] E. Braverman and R. Mamdani, "Continuous versus pulse harvesting for population models in constant and variable environment," Journal of Mathematical Biology, vol. 57, no. 3, pp. 413-434, 2008.

[12] D. Ludwig, "Harvesting strategies for a randomly fluctuating population," Journal du Conseil pour l'Exploration de La Mer, vol. 39, pp. 168-174, 1980.

[13] C. Xu, M. S. Boyce, and D. J. Daley, "Harvesting in seasonal environments," Journal of Mathematical Biology, vol. 50, no. 6, pp. 663-682, 2005.

[14] S. Sinha and S. Parthasarathy, "Unusual dynamics of extinction in a simple ecological model," Proceedings of the National Academy of Sciences of the United States of America, vol. 93, no. 4, pp. 15041508, 1996.

[15] N. P. Chau, "Destabilising effect of periodic harvest on population dynamics," Ecological Modelling, vol. 127, no. 1, pp. 1-9, 2000. 
[16] L. Berezansky and E. Braverman, "On impulsive Beverton-Holt difference equations and their applications," Journal of Difference Equations and Applications, vol. 10, no. 9, pp. 851-868, 2004.

[17] S. Tang, R. A. Cheke, and Y. Xiao, “Optimal implusive harvesting on non-autonomous Beverton-Holt difference equations," Nonlinear Analysis: Theory, Methods \& Applications, vol. 65, no. 12, pp. 2311-2341, 2006.

[18] Z. AlSharawi and M. Rhouma, "Coexistence and extinction in a competitive exclusion Leslie/Gower model with harvesting and stocking," Journal of Difference Equations and Applications, vol. 15, no. 11-12, pp. 1031-1053, 2009.

[19] Z. AlSharawi and M. Rhouma, "The Beverton-Holt model with periodic and conditional harvesting," Journal of Biological Dynamics, vol. 3, pp. 463-478, 2009.

[20] C. W. Clark, Mathematical Bioeconomics, the Optimal Management of Renewable Resources, Pure and Applied Mathematics, John Wiley \& Sons, New York, NY, USA, 2nd edition, 1990.

[21] J. M. Cushing and S. M. Henson, "A periodically forced Beverton-Holt equation," Journal of Difference Equations and Applications, vol. 58, no. 12, pp. 193-210, 1998.

[22] S. Elaydi and R. J. Sacker, "Nonautonomous Beverton-Holt equations and the Cushing-Henson conjectures," Journal of Difference Equations and Applications, vol. 11, no. 4-5, pp. 337-346, 2005.

[23] S. J. Schreiber, "Allee effects, extinctions, and chaotic transients in simple population models," Theoretical Population Biology, vol. 64, no. 2, pp. 201-209, 2003.

[24] S. J. Schreiber, "Chaos and population disappearances in simple ecological models," Journal of Mathematical Biology, vol. 42, no. 3, pp. 239-260, 2001.

[25] J. M. Cushing and S. M. Henson, "Global dynamics of some periodically forced, monotone difference equations," Journal of Difference Equations and Applications, vol. 7, no. 6, pp. 859-872, 2001.

[26] V. L. Kocic, "A note on the nonautonomous Beverton-Holt model," Journal of Difference Equations and Applications, vol. 11, no. 4-5, pp. 415-422, 2005.

[27] Z. AlSharawi, "Periodic orbits in periodic discrete dynamics," Computers \& Mathematics with Applications, vol. 56, no. 8, pp. 1966-1974, 2008.

[28] Z. AlSharawi, J. Angelos, S. Elaydi, and L. Rakesh, "An extension of Sharkovsky's theorem to periodic difference equations," Journal of Mathematical Analysis and Applications, vol. 316, no. 1, pp. 128-141, 2006.

[29] E. Braverman and S. H. Saker, "On the Cushing-Henson conjecture, delay difference equations and attenuant cycles," Journal of Difference Equations and Applications, vol. 14, no. 3, pp. 275-286, 2008.

[30] G. Woeginger, "When Cauchy and Holder met Minkowski: a tour through well-known inequalities," Mathematics Magazine, vol. 82, pp. 202-207, 2009. 